\title{
Application and Research of Man-Machine Interface and Communication Technique of Mobile Information Acquisition Terminal in Facility Production
}

\author{
Jinlei $\mathrm{Li}^{1{ }^{1,},}$ Xin Zhang ${ }^{1}$, Quanming Zhao ${ }^{1}$, Wengang Zheng ${ }^{2}$, \\ Changjun Shen ${ }^{2}$, and Zhipeng $\mathrm{Shi}^{2}$ \\ ${ }^{1}$ Beijing Research Center of Intelligent Equipment for Agriculture, Beijing 100097, China \\ ${ }^{2}$ Key Open Laboratory for Agricultural Information Technology of Ministry of \\ Agriculture, Beijing 100097, China
}

\begin{abstract}
As long as the deepen application of the Object Network Technology in the facility construction, a hot topics in research is coming out, that is how to achieve mobile operation through convenient and high-efficiency multiple resources utilization, in order to increase the proportion of the efficiency and output for the high tech facility in manufacture. The mobile collection terminals could display, transfer and collect the information from wireless sensor network high efficiency. This terminal will play an important role in the Object Network Technology application system, comparing to the others, it has such advantages of the good man-machine interaction (MMI) and reliable communication technology. We develop a mobile information collection terminal basic on the wireless sensor network, the information collection technology adopts the APC240 module to realize the wireless sensor data collection by the MODBUS Agreement; adopt wireless communication module to transfer the GPRS data; MMI realize the screen touch operation, also transplant the uC/OS+GUI making the operation more simply and understandably. This system is much convenience for more rural people to operate in daily work and with more application in agricultural construction in the future.
\end{abstract}

Keywords: facility production, man-machine interface, communication technique, mobile acquisition terminal, GPRS.

\section{$1 \quad$ Preface}

In resent years ,the Internet of things technology and equipment to be promoted and applied in the field of facilities agriculture, including the collection of temperature, humidity, illumination and other environmental information, to predict scientifically

\footnotetext{
* Fund Projects.

The National 863 project : The Accurate Control Technology and Products of Agriculture Efficient Water Use (2011AA100509).

The science and technology program of Beijing: The Integration of Object Network Technology in Agriculture (D111100001011002).
} 
though network transmission, to improve the agricultural comprehensive benefit through helping farmers to cultivate scientifically, the combination of Internet of things and facilities agriculture is a present direction of advanced facilities agriculture[1,2]. The following problems are in the specific application process: A The ways of agriculture acquirement is single and difficult to achieve the mobile operation, B The agricultural environment information transmission technology is not flexile enough, the routing is complex and maintaining is difficult, C Most manmachine interfaces (MMI) on control cabinet are not friendly that additional training is needed and unnecessary loss caused by improper operation often occurs[3]. Developing mobile information collection terminal can make man-machine interfaces and agriculture facilities combined and On-side environmental control equipment and remote monitoring center connected.

The communication technology plays an important role in the facilities agriculture, which including wired communication and wireless communication. It's known that facilities agriculture has the characteristics of object diversity, broad region, remote distribution, etc. And the communication condition is relatively backward, the last mile bottleneck phenomenon highlights in the agriculture information and communication. The wiring of wired communication is difficult in facilities agriculture, and sometimes can not be achieved, it will require a considerable investment if using the wired mode, and the distance of wired communication is relatively short, that unable to achieve remote monitoring. In resent years, the use of wireless communication has became increasing prevalent in facilities agriculture, such as distributed greenhouse monitoring controlling system based on Bluetooth technology, the monitoring system based on GSM and wireless sensor network, the above systems have good equipment interoperability, strong anti-jamming capability and good real-time performance,etc.,but some wireless communication technologies have poor stability, low reliability, high loss of power and lack transmitted distance, etc..[6].

To compare the advantage and disadvantage of WSN and GPRS communication technology, we combine with the advantages of good real-time performance low power loss from WSN technology and far transmission distance from GPRS technology, also covering shortages of each other, through designing a communication system being suitable to the Agricultural environment work. The operators of mobile information collection terminal is agricultural employee, it request a simple operation interface, also with the instruction function to make the communication between operator and equipment more smoothly which increase the efficiency. We state a MICT with low power loss, low cost, stabile communication and simple operate through the combination of Object internet communication and facilities agriculture to solve the disadvantages in daily works, such as complex control, high cost for cable arrangement and poor mobile information collection performance. 


\section{System Structure and Function}

Regarding to daily application, the structure of this system is designed basic on agricultural wireless sensor network facilities for agricultural production mobile information collection system. Essentially, the system structure is usually shown as that in Figure 1.

The typical working way of this system is: A variety of wireless sensor nodes are laid in the collection area according to the needs of farmers, each node could collect the different agro-environmental information through the sensor probe. At the same time, a perception network (WSN) shall rapidly be constructed through selforganization between the nodes. The sensor data collected by the sensor nodes shall be transmitted to the mobile information collection terminals by multi-hop relay. Communication between the mobile information collection terminals and management and user networks need realize the WAN interconnection via GPRS. The mobile information collection terminals act as a bridge of communication between the perception network and the WAN. The perception of agricultural environmental data collected by the network shall be delivered to the WAN via GPRS and the remote monitoring shall be carried out by the end farmer.

The mobile collection terminals not only have the gateway function but also have the node management, data management and other functions in wireless sensor networks. The collection area includes the wireless sensor nodes of a variety of information, so need a unified node management, such as configuration of sensor node ID, node location, sampling period, node power and other properties.

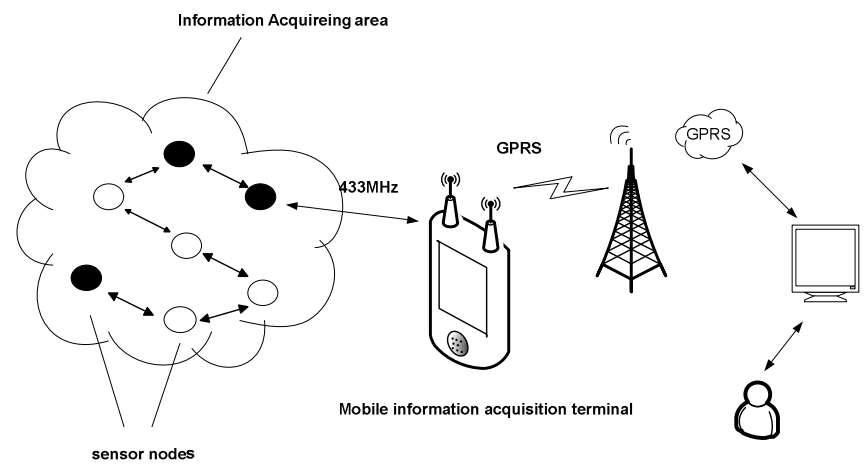

Fig. 1. Data management refers data storage display and other management to data of wireless senor network that the mobile collection terminals periodically collected

\section{$3 \quad$ Hardware Design of Mobile Acquisition Terminal}

The terminal includes mainly six parts: microprocessor module(MSP430F5438), GPRS module, APC240 module, man-machine interface module, memory module and power module. APC240 module is used to collect and manage the wireless sensors network data through $433 \mathrm{MHz}$ public frequency. Remote transmission module use SIM900A to send the information which is collected by APC240 module. The system structure is shown as that in Figure 2. 


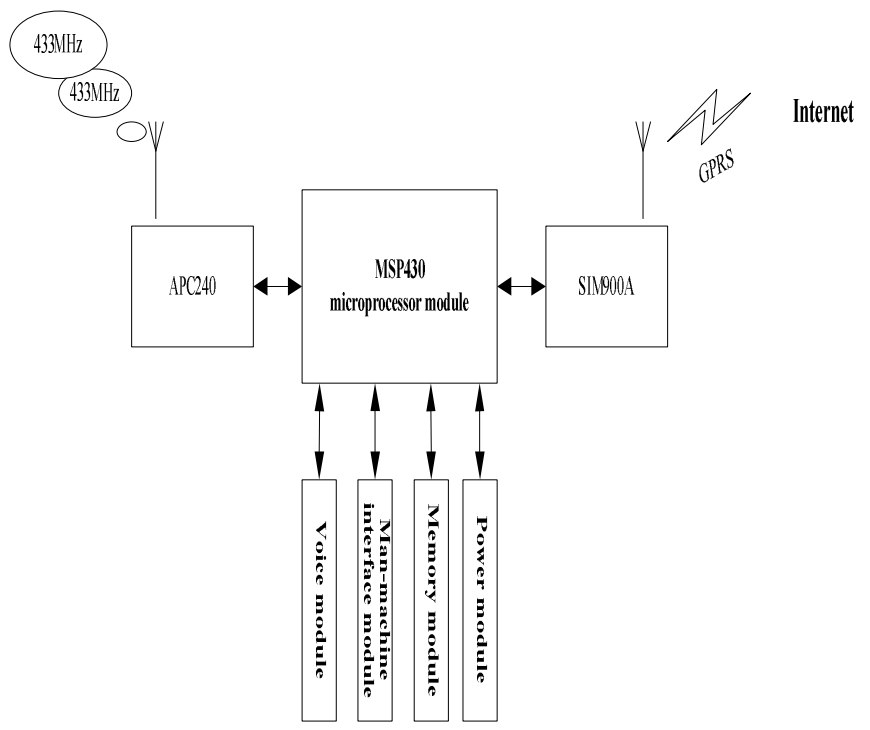

Fig. 2. Overall block diagram of system. The farmers could monitor the environmental information with the wireless temperature and humidity measurement system software.

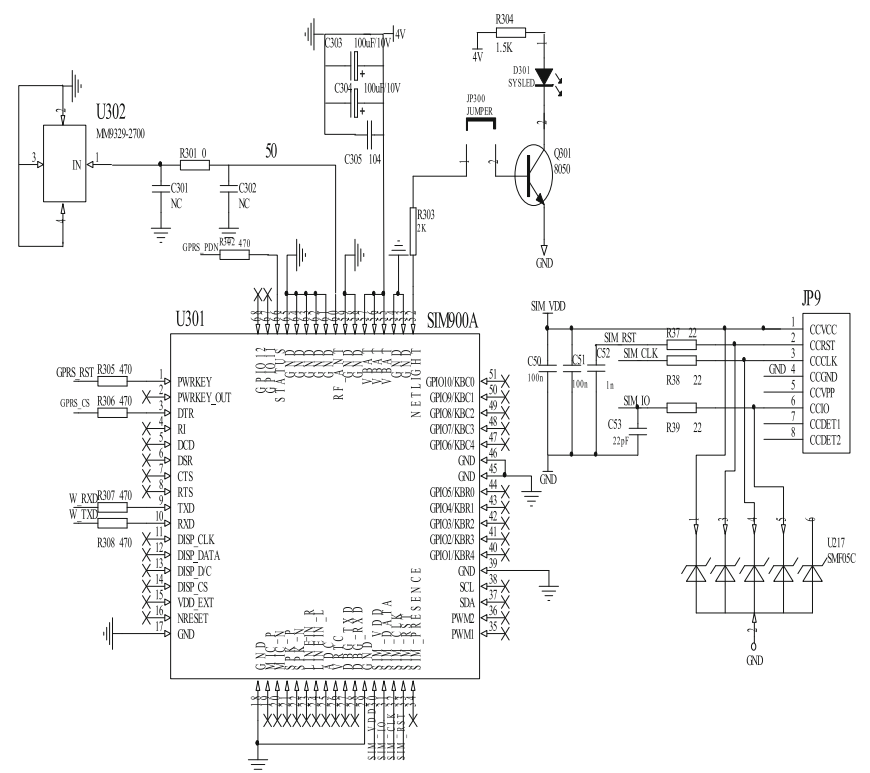

Fig. 3. U301 is the SIM900A module. The microprocessor (msp430) sends AT instructions to SIM900A module with the serial interface (W_RXD,W_TXD); JP9 is the socket of SIM card module. SIM card communicates with SIM900A through SIM_RST SIM_CLK and SIM_IO.U302 is the antenna of SIM900A module. 
This system uses the SIMcom company's GPRS module SIM900A,which contains built-in TCP/IP protocol and TCP/IP AT instructions. SIM900A provides Antenna interface, Asynchronous serial interface and SIM card interface. The circuit structure of this module is shown as that in Figure 3.

\section{The Software Design}

\subsection{The Design of Man-Machine Interface}

The main interface includes mainly four parts, Time-setting sub-interface, Historical data sub-interface, Data collection sub-interface and system shutdown sub-interface. The function of time-setting sub-interface is that displaying time and setting time; in addition to display historical data, the historical data sub-interface can delete or store the sensor data; the farmers can shut down the terminal as need through the system shutdown sub-interface; the data collected by wireless sensors will be showed on data collection sub-interface. The system Man-machine Interface structure is shown as that in Figure 4.

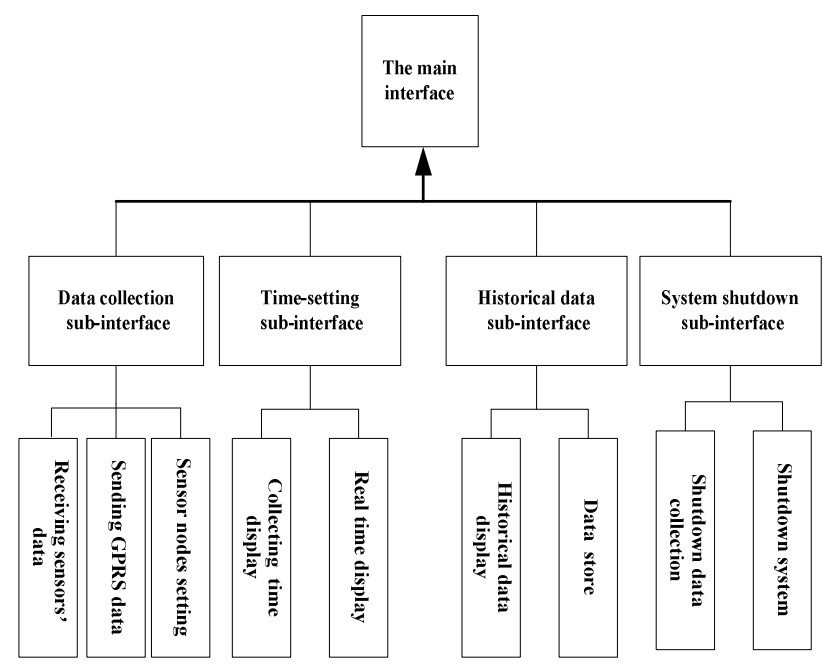

Fig. 4. Our design for MMI interface adopt the mature UC/OS+GUI technology, the operator click on the lively and visual icon but no need to input the number and characters, then it become simple, natural and friendly between man and machine.

\subsection{The Design of Communication Module}

The communication module includes mainly two parts: GPRS Transmission and the collection of wireless sensor network. The main work about the communication module is that setting different nodes addresses according to different sensors. 
During the information transmission, the first thing is to initialize the SIM900A including the baud rate setting and mobile mode closure,etc. The key instructions are showed as follows:

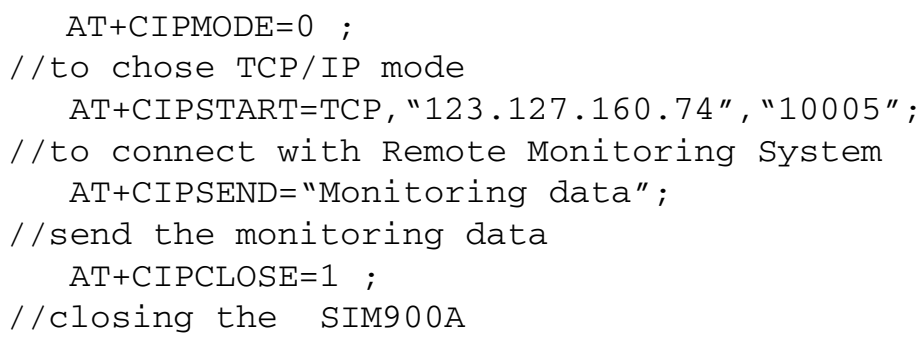

The PC software of remote monitoring center "wireless temperature and humidity collection system" could help farmers to monitor the real-time environmental information in farmland, which is developed to collect temperature and humidity data basic on the VS 2008 PLATFORM C\#. Many node equipments could be connected through this software for monitoring environmental data ,such as air temperature, air humidity and soil temperature, etc. Also it could lead out the data form format through such terminal.

\subsection{Implementation and Call of Main Task in System}

In order to enhance the system's real-time, ucGUI multi-task system is applied and a UC/OS-II core is transplanted into MSP430 while the following content changes:

MSP430 stack member is 16, so the CPU_STK declared as unsigned integer data type. MSP430 stack grows from top to bottom. When OS_STK_GROWTH is defined as 0, it said the stack grows from bottom up, and when OS_STK_GROWTH is defined as 1 , it said the stack grows from top down.

Typedef unsigned int

\#define OS_STK_GROWTH

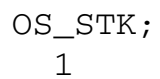

Transplantation of ucGUI mainly includes modification on three configuration files in the GUI/Config directory and writing of touch screen drive. LCDConf.h configuration file defines LCD control register, the display screen size and other optional features.

During normal operation of the system, Interrupt Service Routine ISR provides some related services of multi-task operating system, such as semaphore and mailbox ,etc., the flow chart of system main software is shown as that in Figure 5. 


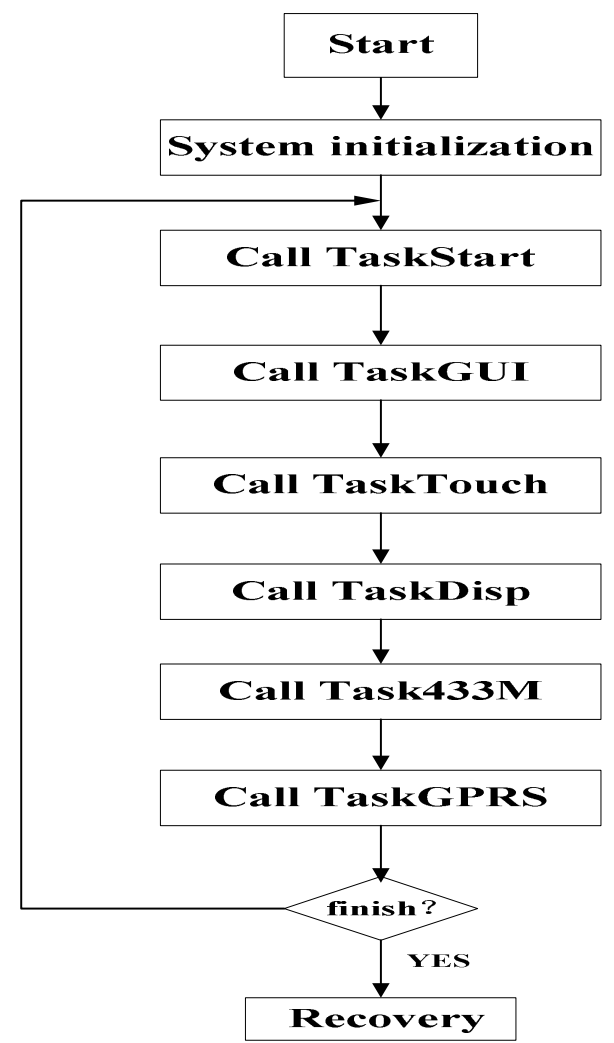

Fig. 5. The system uses six tasks, from highest to lowest priority order: TaskStart, TaskGUI, TaskTouch, TaskDisp, Task433M, TaskGPRS, in which the TaskStart is created in main function, the other five tasks are created in the TaskStart.

\section{Summarization}

Through a wholly analysis to the actual demand of current facilities agriculture, then get a research of the MMI interface of mobile information collection terminals in facilities construction and communication technology. The design of mobile information collection terminals mentioned here have three advantages as follows: 1.Combining the GPRS technology and wireless sensor network makes the collection terminal does not rely on the wireless sensor and realize the real-time efficiency of remote monitoring system more stronger 2 . The wireless communication module solve the complex cable arrangement limitation and realize the long-way transmission whenever and wherever 3. Successfully remove the UC/GUI format software, making the MMI more lively and simply, also solving the problems during operations to the complex control system, then low down the cost, more practically. 


\section{References}

1. Pan, J.: The Object Network and Facility Agriculture. Agriculture Machinery Technology Extension (2), 45 (2010)

2. Zhou, X.: Facility Agriculture Online Monitoring System Based on Internet of Things. Journal of Taiyuan University of Science and Technology (2), 12 (2011)

3. Jia, X., Wang, C., Qiao, X.: Drive of wireless smart terminal and touch-screen humanmachine interface for field irrigation 9, 8-12 (2008)

4. Ayday, C., Safak, S.: Application of Wireless Sensor Networks with GIS on the Soil Moisture Distribution Mapping, Ostrava, 25. - 28.1. (2009); Pu, Z.H. B.: Photo electricity testing technology. Machinery Industry Press, Beijing (2005)

5. Sun, Z., Zhao, W., Liang, J., Du, K., Zhang, Y.: Design and application of greenhouse remote automatic control system based on GPRS and WEB. Control \& Automation (2010)

6. Texas Instruments. MSP430X5XX User's Guide (2008) 